
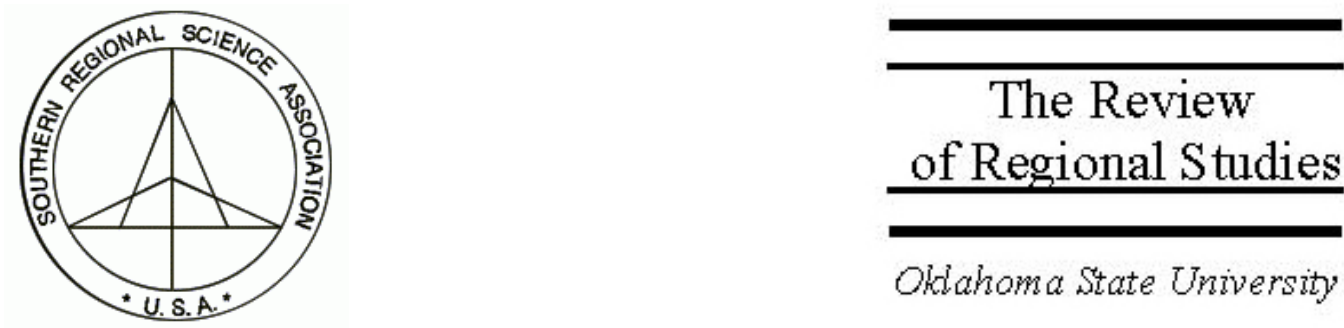

Oklahoma State University

\title{
Using Ready-Made Regional Input-Output Models to Estimate Backward-Linkage Effects of Exogenous Output Shocks
}

\author{
Scott R. Steinback \\ National Marine Fisheries Service, Northeast Fisheries Science Center, Social Sciences \\ Branch, 166 Water Street, Woods Hole, Massachusetts 02543-1097, \\ e-mail: scott.steinback@noaa.gov
}

\begin{abstract}
The Leontief input-output model provides the basis for quantifying backward-linked multiplier effects of exogenous final demand shocks. In certain situations, however, policies or uncontrollable factors induce exogenous changes in gross industry output. Application of the usual Leontief multipliers in these cases will lead to biased calculations of backward-linked economywide effects. To eliminate this bias, an output-based adjustment method is proposed that relates demand-driven Leontief multipliers to exogenous output shocks. It is demonstrated that this approach yields exactly the same results as the mixed exogenous/endogenous variables technique, a commonly employed adaptation procedure that accepts outputs as entries. The equivalence between these two approaches is important because the output-based approach offers substantial computational savings through ease of implementation within ready-made regional input-output systems and provides practitioners with the ability to generate disaggregated estimates of indirect multiplier effects.
\end{abstract}

Keywords: Ready-made input-output models; Demand-driven; Exogenous output shocks, Regional purchase coefficient; Policy appraisals 


\section{INTRODUCTION}

The Leontief input-output model, which accounts for both direct economic effects and backward-linked multiplier effects, provides a convenient framework for quantifying the short-run economy-wide effects of final demand shocks. As known, the final demand elements are considered exogenous, and it is the effects of changes in these elements on endogenously determined gross outputs that are quantified through the standard Leontief model. However, there are certain regional input-output modeling scenarios, such as when the productive capacity of a sector is curtailed because of a policy action, that require predetermination of sectoral output rather than simultaneous determination by final and intermediate demand. Application of the usual Leontief multipliers in such a case will lead to biased calculations of backward-linked economy-wide effects because the level of sectoral output is not determined by demand but by capacity constraints. The above-mentioned capacity reduction scenario is just one example of the more general scenario in which sectoral production and its expenditure pattern is, in the short-run at least, restricted to some predetermined level. The more general scenario includes those cases where output is reduced because of policy changes (Roberts 1994; Waters, Holland, and Weber 1994; Natcher, Geenberg, and Herrmann 1999; National Marine Fisheries Service 2001; and Leung and Pooley 2002), weather conditions (Hoppe 1978), depletion of raw materials (Petkovich and Ching 1978), and labor strikes (Miller and Blair 1985, p. 325). In contrast, it also includes those situations where output is increased because of regional shifts in sectoral production (Marcouiller, Schreiner, and Lewis 1996), irrigation expansion (Findeis and Whittlesey 1984), or reallocation of resources across sectors (Eiser and Roberts 2002).

The primary objective of this study was to develop an input-output approach capable of estimating the backward-linked region-wide effects of any case in which a change in sectoral output is predetermined. Attempts to resolve the problem of handling constraints on sectoral output include the development of an iterative linear programming solution applied to the input-output model (Petkovich and Ching 1978). This procedure provides a solution for one case of the more general scenario that involves output predetermination for any industry sector in the model. To accommodate the more general scenario, a procedure was developed that explicitly transforms the traditional Leontief model into a model that is driven by a change in the demand for inputs arising from a predetermined change in gross output (Johnson and Kulshreshtha 1982). This latter technique, now commonly referred to as a mixed exogenous/endogenous variables model (Miller and Blair 1985, p. 325), allows for the incorporation of all cases where a change in sectoral output is predetermined and is particularly useful for relative aggregate policy appraisals of changing resource management strategies. In Eiser and Roberts (2002), for example, policy-driven shifts in forest expansion away from coniferous plantations towards broadleaf and native species are translated into predetermined output changes within a mixed exogenous/endogenous variables model, and the resulting relative impacts on the aggregate economy are compared across four different woodland planting scenarios. Appraisals such as this are important to resource managers because they provide 
aggregate information about the relative impacts of management alternatives that can be used to make more informed regional policy decisions.

In addition to considering the total impacts of a predetermined output change in aggregate, however, natural resource policymakers also want to know how the impacts will be dispersed throughout a particular region in as much detail as possible. Unfortunately, the sectoral detail in regional mixed exogenous/endogenous variables models is often limited due to computational complexities, so it is difficult to derive disaggregated estimates of indirect multiplier effects. In part, this is because all of the existing readymade commercial regional input-output models, such as IMPLAN, RIMS II, and RSRI's PC I-O, which provide considerable sectoral detail to trace backward linkages, are based on traditional Leontief techniques and it is not possible to incorporate the modified relationships embodied in the mixed exogenous/endogenous variables approach into these ready-made models. As a consequence, most of the industry sectors in a regional mixed exogenous/endogenous variables model are frequently derived from condensed versions of available input-output models and lack the sectoral detail necessary for fully informed decision making (see Eiser and Roberts 2002; and Leung and Pooley 2002).

In an attempt to provide a higher level of sectoral detail and to minimize computational complexity, it will be demonstrated that with adjustments to the regional purchase coefficients, standard Leontief techniques can be employed within ready-made inputoutput models to translate predetermined output shocks into backward-linked economywide effects. This output-based adjustment approach yields exactly the same aggregate solution as the mixed exogenous/endogenous variables technique; but since it takes advantage of the sectoral detail inherent in ready-made input-output models, it also provides disaggregated estimates of indirect multiplier effects. Furthermore, the approach offers substantial computational savings through its ease of implementation within ready-made regional input-output models and its mathematical familiarity to traditional Leontief techniques.

The output-based adjustment model derived in this paper, however, does of course suffer from the better-known restrictions of input-output analysis such as fixity of prices and zero-substitution elasticities in consumption and production. In addition, since the output-based multipliers measure only the backward-linked impacts of predetermined output changes, the forward effects on the production of sectors that utilize the exogenized outputs as inputs are not explicitly considered. Thus, the approach is most suitable for modeling capacity constraint scenarios where there are limited purchases of the exogenized sector(s) outputs by the other endogenous sectors in the model. An example of this scenario is found in Eiser and Roberts (2002) where there are significant backward-linked effects in the region generated from policy-driven changes in the level of woodland planting, with rather limited flows of impacts from the maintenance sector and no impacts in the short-run from the harvesting and processing sectors due to a production cycle that reaches 100 years. Similarly, if much of the output of an exogenized sector(s) is exported out of the region, which is frequently the case in the production of natural resources (e.g., mining, fisheries, and timber) and farm products, especially in 
small regional input-output models, the output-based approach derived here would capture the majority of the short-run regional impacts generated from a capacity constraint change. ${ }^{1}$

The paper is structured as follows. In the next section, the nature of the bias that results if traditional Leontief multipliers are used to estimate the effects of capacity constraint changes is explored in more detail. Section 3 provides a brief review and derivation of the input-output model most frequently employed in the literature when a change in sectoral output is predetermined and not simultaneously determined by final and intermediate demand, the mixed exogenous/endogenous variables model. Section 4 describes the main innovation of the paper, the output-based adjustment procedure, which translates exogenous output shocks into backward linked economy-wide effects; and it is shown that this adjustment method yields exactly the same solution as the mixed exogenous/endogenous variables approach. Finally, Section 5 summarizes the findings and provides some concluding remarks regarding model assumptions and limitations.

\section{OVERESTIMATION OF ENDOGENOUS EFFECTS}

The traditional Leontief input-output approach derives sectoral outputs from exogenously specified final demands as

$$
X=(I-A)^{-1} * \hat{Y}
$$

where $X$ is a $\mathrm{n} \times 1$ column vector denoting output, $I$ is a $\mathrm{n} \times \mathrm{n}$ identity matrix, $A$ is a $\mathrm{n} \times \mathrm{n}$ direct input coefficient matrix, and $\hat{Y}$ is a $\mathrm{n} \times 1$ column vector denoting exogenous final demand. The elements of $A\left(a_{i j} s\right)$ are called direct input coefficients and are denoted as

$$
a_{i j}=\frac{z_{i j}}{X_{j}}
$$

where $z_{i j}$ is the level of sales from sector $i$ to sector $j$, and $X_{j}$ is the total output of sector $j$. The $(I-A)^{-1}$ matrix is often referred to as the Leontief inverse, comprised of the interdependence coefficients $\left("{ }_{i j} \mathrm{~s}\right)$, designated here as "industry specific" output

\footnotetext{
${ }^{1}$ It is important to note that the input-output model of Ghosh (1958) has been used in applications to examine potential forward linkage effects of capacity constraint changes (see Papadas and Dahl 1999; Eiser and Roberts 2002; and Leung and Pooley 2002). However, as pointed out in these applications and critiqued by Oosterhaven (1988), Gruver (1989), and Oosterhaven (1989), the theoretical interpretation of the forward linkage effects using the Ghosh framework is problematic, and any casual interpretation or application will probably lead to nonsensical results. As such, no attempt was made to incorporate the Ghosh framework into the derived output based approach. Computable general equilibrium (CGE) models have the potential to address some of these limitations by incorporating market-equilibrating mechanisms. However, CGE models are more data intensive and generally necessitate even greater sectoral aggregation than is required in the construction of mixed exogenous/endogenous variables models.
} 
multipliers. These multipliers indicate how much the output of each row sector would change if the final demand for sector $j$ 's output changed by one dollar.

From equation (1) it is clear that the industry-specific multipliers may only be multiplied by exogenous final demand and not with exogenous gross output (a variable that is endogenous in equation 1). If a change in gross output for a particular sector $j$ was treated "as if" it was an exogenous final demand change and multiplied by the industryspecific multipliers, the economy-wide effects would be overestimated. The magnitude of the overestimation would depend upon the level sector $j$ 's "interconnectedness" in the model. The larger the proportion between the sum of the endogenous flows and final demand flows in the model for sector $j$ 's output, the greater the overestimation error. This can be measured by sector $j$ 's on-diagonal element in $(I-A)^{-1}$ (designated as " ${ }_{j j}$ ), which measures the total direct and indirect demand for sector $j$ 's output generated by the exogenous final demand change. Jeong (1984) and Szyrmer (1992) demonstrate that the level of overestimation is a constant percentage calculated from the indirect component of a particular sector's " ${ }_{j j}$ entry. For example, if sector $j$ 's on-diagonal element was 1.0904 and the effect of a change in the gross output of sector $j$ was estimated by applying the standard industry-specific multipliers, the results would overestimate the output impact by 9.04 percent. $^{2}$

\section{MIXED EXOGENOUS/ENDOGENOUS VARIABLES MODEL}

The mixed exogenous/endogenous variables technique was designed to eliminate the overestimation bias and accommodate any scenario that involved output predetermination. This modeling approach explicitly transforms the traditional Leontief model into a model that is driven by a change in the demand for inputs arising from an exogenously specified change in gross output. ${ }^{3}$ The basic premise underlying the model design is that the traditional Leontief model must be modified so that gross output changes can be treated as exogenous, as opposed to only final demands.

In the usual form of standard demand-based models, sectoral outputs are derived from exogenously specified final demands as in equation (1). In a mixed exogenous/ endogenous variables model, some of the sectoral outputs are assumed to be exogenous so that the outputs of the remaining sectors can be quantified endogenously through the

\footnotetext{
${ }^{2}$ As a general rule, one can expect high " ${ }_{j j}$ values for highly aggregated models, models that are closed with respect to households, or models that cover large geographic regions (Lahr and Miller 2001). The feedback effects measured by the ${ }_{j j}$ element become less intense for highly disaggregated models that cover small geographic regions. Therefore, as the level of openness increases, " ${ }_{j j}$ elements approach unity and the bias introduced by applying the standard industryspecific multipliers to gross output shocks decreases.

${ }^{3}$ The terminology used in this paper follows that used in Miller and Blair (1985). Papadas and Dahl (1999) and others refer to the mixed exogenous/endogenous variables model as a backward supply-driven model and as they (Papadas and Dahl) point out, readers should not confuse this backward-linked approach with the input-output model of Ghosh (1958), often also referred to a "supply-driven," and from which forward-linked multipliers are derived.
} 
model. The basic relationships embodied in the demand-based approach are the same, but the equations that comprise $(I-A) * X=Y$ are rearranged in order to isolate the exogenous variables on the right-hand side and the endogenous variables on the left. For example, in a two-sector economy, if $X_{1}$ and $Y_{2}$ are treated as exogenous, the equations of the system can be written in matrix notation as

$$
\left[\begin{array}{cc}
-a_{12} & -1 \\
\left(1-a_{22}\right) & 0
\end{array}\right]\left[\begin{array}{c}
X_{2} \\
Y_{1}
\end{array}\right]=\left[\begin{array}{cc}
-\left(1-a_{11}\right) & 0 \\
a_{21} & 1
\end{array}\right]\left[\begin{array}{c}
\hat{X}_{1} \\
\hat{Y}_{2}
\end{array}\right]
$$

If the matrix of elements on the left-hand side of equation (2) is denoted $M$ and the matrix of elements on the right-hand side $N$, then the solution will be of the form

$$
\left[\begin{array}{c}
X_{2} \\
Y_{1}
\end{array}\right]=M^{-1} N\left[\begin{array}{l}
\hat{X}_{1} \\
\hat{Y}_{2}
\end{array}\right]
$$

In this form, the matrix $M^{-1} N$ reveals industry-specific multipliers that relate the exogenously determined values ( $\hat{X}_{1}$ and $\left.\hat{Y}_{2}\right)$ to those quantified through the model $\left(X_{2}\right.$ and $\left.Y_{1}\right)$. In the derivation of these multipliers, when the isolation of impacts of exogenous output shocks are the objectives of analysis, one can assume zero changes in exogenously specified final demands (Papadas and Dahl 1999). Thus, if $\hat{Y}_{2}$ is set to zero, the suggested multiplier matrix from (3) stems from exogenous output changes and can be used to determine the impact of an exogenously specified $\hat{X}_{1}$ on $X_{2}$. The solution procedure is the same for any square set of linear equations, so expansion to an $n$-sector economy comprised of exogenous output shocks for $k$ sectors (where $k<n$ ) would be calculated in the same manner.

For each of the exogenized sectors in $M^{-1} N$, the column sum of the industry-specific multipliers indicates the total indirect and induced multiplier effects on all other sectors of the economy required to satisfy a one-dollar change in the outputs of each of the exogenized sectors.

The total output effect, however, would also include the direct multiplier effects associated with the initial exogenous change and is easily calculated by simply adding 1 to the column vector sum of the exogenized sector(s). This is because the immediate effect of an exogenous change in a sector's output is directly felt by that particular sector (i.e., the sector's production has to accommodate the exogenous change), which implies a direct multiplier value of 1 .

Overestimation of endogenous effects are avoided by employing a mixed exogenous/ endogenous variables model because there are no multiplier effects associated with inter- 
mediate purchases from the exogenized sector(s). However, as mentioned in the first section, this procedure can be computationally difficult to implement and is limited in its ability to provide detailed estimates of indirect multiplier effects. This is primarily due to the functional form changes required to transform the traditional $A$ matrix into inputoutput tables capable of accepting gross output changes as entries and the inability to incorporate these changes into ready-made models that offer significant sectoral detail, but are based on traditional Leontief relationships. The next section will show that traditional Leontief relationships need not be abandoned in the assessment of capacity constraint changes and that with simple adjustments, ready-made input-output models can accept gross output changes as entries without overestimating the endogenous effects.

\section{OUTPUT-BASED ADJUSTMENT MODEL}

Tanjuakio, Hastings, and Tytus (1996) proposed to eliminate the overestimation bias introduced by assuming that a predetermined change in gross output can be modeled as a change in final demand within a regional Leontief model, by setting the regional purchase coefficients (RPCs) for the outputs produced by the directly impacted industries to zero. An RPC for a particular industry sector's output indicates the portion of the total regional demand supplied by the local producers. Setting the RPCs to zero for the outputs produced by the industries directly impacted effectively prevents other local industries from buying these outputs. ${ }^{4}$ Since these are the endogenous effects that are the source of the inherent overestimation problems, the bias is eliminated.

Algebraically, when the RPC for the output produced by sector $i$ is set to zero, the row elements for sector $i$ in the $A$ matrix $\left(a_{i j} s\right.$ ) become zero, and since $(I-A)^{-1}$ is derived by subtracting the $A$ matrix from the identity matrix, the ${ }_{j j}$ element for sector $i$ in the new Leontief inverse becomes one while the remaining row elements are forced to zero. Therefore, multiplication of an exogenous change in the gross output of sector $j$ (where $i=j$ ) by the adjusted row elements in sector $i$ will produce the initial exogenous change, regardless of the origin of the change. By definition, since the modified industry-specific multiplier for sector $i$ is one where $i=j$ (designated herein as $\bar{\alpha}_{j j}$ ) and zero where $i . . j$,

\footnotetext{
${ }^{4}$ In this paper, the implied assumption is that there is only one RPC value for each commodity produced by a given sector. Because of the many difficulties involved in characterizing regional commodity demand on an industry-by-industry basis, ready-made input-output models typically only provide these uniform average values. Through primary data collection, however, it may be possible to obtain a full matrix of RPCs. This, of course, would then require one to set all of the RPCs for each commodity produced by the directly impacted sector(s) to zero. Additionally, for simplicity, it is assumed that each industry sector produces a single homogeneous commodity. In addition to primary commodities, however, many industries produce additional commodities known as "secondary" commodities. To fully eliminate purchases from an industry sector that produces secondary products, it may be necessary to set the RPCs of the secondary products to zero as well.
} 
there are no intermediate demands for sector i's output in the modified Leontief inverse. ${ }^{5}$ Additionally, since $\bar{\alpha}_{j j}$ is set to one, the remaining modified industry-specific multipliers in that column $j$ of $(I-\bar{A})^{-1}$ indicates how much the output of each row sector would change if the output of sector $j$ changed by one dollar. Recall that in the traditional final demand-based input-output approach, these multipliers specify how much the output of each row sector would change if the final demand for sector $j$ is changed by one dollar. Thus, using the modified output-based approach in which the RPCs for the outputs produced by the directly impacted industries are set to zero alters the traditional interpretation of the industry-specific multipliers. In fact, in an $n$-sector economy, given a vector of final demands comprised of exogenous changes for $k$ sectors (where $k<n$ ) and zeros for the remaining $n-k$ sectors, equation (1) can be rewritten as

$$
X=(I-\bar{A})^{-1} * \hat{Y}
$$

where $\bar{A}$ represents the new $\mathrm{n} \times \mathrm{n}$ direct input coefficient matrix when the RPCs for the outputs produced by the $k$ exogenous sectors are set to zero. If the vector $\hat{Y}$ is set equal to the exogenous gross output changes for the $k$ sectors, the economy-wide impacts can be generated through postmultiplication of the modified industry-specific multipliers in $(I-\bar{A})^{-1}$ by the exogenous changes contained in vector $\hat{Y}$ without exaggerating the endogenous effects. ${ }^{6}$ Readers should note that the total output multiplier for each $k$ sector will be greater than one because it includes the modified on-diagonal industry-specific multiplier, which is set to one (i.e., direct effect), and the indirect and induced multiplier effects on output required from all $n-k$ sectors in the economy.

Given that industry-specific multipliers derived from this approach relate exogenous gross output shocks to backward-linked economy-wide impacts, the multipliers should match those generated from a mixed exogenous/endogenous variables model. Indeed, this is the case, and for ease of illustration is best represented by a two-sector example. Consider a two-sector $(I-A)$ matrix in which the level of change in $X_{1}$ is treated as exogenous. If the RPC for the output produced by sector 1 is set to zero, the $(I-A)$ matrix becomes

\footnotetext{
${ }^{5}$ Groenewold, Hagger, and Madden (1987) point out that setting the elements of a particular row in an A matrix to zero is equivalent to that sector's relocating to outside of the modeling region but continuing to purchase intermediate inputs from the remaining sectors (in the same proportions as prior to relocation).

${ }^{6}$ If the outputs of more than one sector are assumed to be exogenous, however, the effects on the economy should be estimated simultaneously to avoid capturing linkages between the exogenized sectors themselves. If estimated separately, the summation of the effects could capture interindustry linkages between the exogenized sectors, resulting in double-counting and inflated estimates. This proviso also applies to the mixed exogenous/endogenous variables model if more than one sector's output is specified exogenously.
} 


$$
(I-\bar{A})=\left[\begin{array}{cc}
1 & 0 \\
-a_{21} & \left(1-a_{22}\right)
\end{array}\right]
$$

and

$$
(I-\bar{A})^{-1}=\left[\begin{array}{cc}
1 & 0 \\
\frac{a_{21}}{\left(1-a_{22}\right)} & \frac{1}{\left(1-a_{22}\right)}
\end{array}\right]
$$

From equation (4), if $\hat{Y}_{1}$ is set to the exogenous change in $X_{1}$, the vector of output requirements necessitated by the equivalent change in $\hat{Y}_{1}$ can be rewritten in matrix form as

$$
\left[\begin{array}{l}
X_{1} \\
X_{2}
\end{array}\right]=\left[\begin{array}{cc}
1 & 0 \\
\frac{a_{21}}{\left(1-a_{22}\right)} \frac{1}{\left(1-a_{22}\right)}
\end{array}\right]\left[\begin{array}{c}
\hat{Y}_{1} \\
0
\end{array}\right]
$$

The output-based solution vector $X$ is then equal to

$$
\left[\begin{array}{l}
X_{1} \\
X_{2}
\end{array}\right]=\left[\begin{array}{c}
\hat{Y}_{1} \\
\frac{a_{21}}{\left(1-a_{22}\right)} \hat{Y}_{1}
\end{array}\right]
$$

The coefficients of $\hat{Y}_{1}$ in the solution vector represents the modified industry-specific multipliers $\left(\alpha_{i j} \mathrm{~s}\right)$ in column 1 of $(I-\bar{A})^{-1}$. As a result of setting the RPC for $X_{1}$ to zero, there are no intermediate demands for the output of sector 1 , so $\bar{\alpha}_{j j}=1$ and $X_{1}$ equals the initial exogenous change $\left(\hat{Y}_{1}\right)$. The indirect effects are measured from the expression $\frac{a_{21}}{\left(1-a_{22}\right)}$, which indicates how much the output of sector 2 would change if the output of sector 1 is changed by one dollar. The summation of both industry-specific multipliers $\left(1+\frac{a_{21}}{\left(1-a_{22}\right)}\right)$ measures the total direct and indirect multiplier effects in 
the two-sector economy required to satisfy a one-dollar change in the gross output of sector 1 .

Continuing with the same two-sector example, the basic form of the mixed exogenous/endogenous variables model can be written as in equation (2). If the matrix of coefficients on the left-hand side of (2) is denoted $M$ as in equation (3), then $M^{-1}$ gives

$$
M^{-1}=\left[\begin{array}{cc}
0 & \frac{1}{\left(1-a_{22}\right)} \\
-1 & \frac{-a_{12}}{\left(1-a_{22}\right)}
\end{array}\right]
$$

and, following the procedure in (3),

$$
\left[\begin{array}{c}
X_{2} \\
Y_{1}
\end{array}\right]=\left[\begin{array}{cc}
\frac{a_{21}}{\left(1-a_{22}\right)} & \frac{1}{\left(1-a_{22}\right)} \\
\left(1-a_{11}\right)-\frac{a_{12}}{\left(1-a_{22}\right)} a_{21} & \frac{-a_{12}}{\left(1-a_{22}\right)}
\end{array}\right]\left[\begin{array}{c}
\hat{X}_{1} \\
\hat{Y}_{2}
\end{array}\right] .
$$

If $\hat{Y}_{2}$ is set to zero and the interest is only in the impact of an exogenous change in the output of sector 1 , on sector 2 , equation (6) leads to

$$
X_{2}=\frac{a_{21}}{\left(1-a_{22}\right)} \hat{X}_{1}
$$

The expression $\left(\frac{a_{21}}{\left(1-a_{22}\right)}\right)$ represents the indirect output effects on sector 2 of a onedollar change in the output of sector 1 and corresponds exactly to the industry-specific multiplier found in the solution vector for $X_{2}$ using the output-based approach (equation (5)). If the direct output multiplier of 1 is also included, the effect of an exogenously determined $\hat{X}_{1}$ on the outputs of sectors 1 and 2 generates the same value as in equation (5) (since $\hat{Y}_{1}$ is assumed to be equivalent to $\hat{X}_{1}$ in equation (5)). ${ }^{7}$ The same principles

\footnotetext{
${ }^{7}$ An identical solution is also provided if each of the standard Leontief coefficients $\left(\alpha_{i j} \mathrm{~s}\right)$ in the hypothetical two-sector example were divided by their respective on-diagonal elements $\left(\alpha_{j j} \mathrm{~s}\right)$ and
} 
can be used to extend this equivalence to an n-sector model. For the interested reader, a $3 \times 3$ example of the equivalence between the modified output-based approach and the mixed exogenous/endogenous variables approach is provided in the Appendix.

Although the two models yield the same aggregate solution, the modified outputbased approach offers practitioners the ability to provide policy makers with disaggregated estimates of indirect multiplier effects since it can be incorporated into readymade regional input-output systems. By setting the RPCs of the outputs produced by the directly impacted sectors to zero prior to constructing the direct input coefficient matrix (A matrix), and then setting the final demand changes equal to the predetermined gross output shocks, ready-made regional input-output systems can accept gross output changes as entries and be used in a timely manner to partition estimates of aggregate economywide impacts into detailed estimates of indirect multiplier effects.

\section{CONCLUSIONS}

This paper demonstrates the bias that results if traditional Leontief multipliers are used in modeling situations that require predetermination of sectoral output rather than simultaneous determination by final and intermediate demand. A simple adjustment method is proposed that eliminates the bias by relating demand-driven Leontief multipliers to exogenous output shocks. The method is based on traditional Leontief techniques, so it is particularly useful for practitioners who rely on ready-made inputoutput systems to estimate regional input-output tables, compute multipliers, and conduct industry impact assessments. These ready-made systems also offer a high degree of

postmultiplied by the assumed vector of output changes. That is, the matrix of multipliers derived

$$
\begin{aligned}
& \text { from } \alpha_{i j} / \alpha_{j j},\left[\begin{array}{cc}
1 & \frac{a_{12}}{\left(1-a_{11}\right)} \\
\frac{a_{21}}{\left(1-a_{22}\right)} & 1
\end{array}\right] \text {, postmultiplied by }\left[\begin{array}{c}
\hat{X}_{1} \\
0
\end{array}\right] \text {, produces the same solution vector } \\
& {\left[\begin{array}{l}
X_{1} \\
X_{2}
\end{array}\right]=\left[\begin{array}{c}
\hat{X}_{1} \\
\frac{a_{21}}{\left(1-a_{22}\right)} \hat{X}_{1}
\end{array}\right] \text {. This formula was originally provided by Ritz and Spaulding (1975) in }}
\end{aligned}
$$

an unpublished memorandum (addressed to the Bureau of Economic Analysis Interindustry Economic Division Staff), where they explain that “. . . by dividing each coefficient in a column by the main diagonal element, the requirements are related to gross output of the industry rather than its deliveries to final demand" (p. 14). Since then, a variety of interpretations and transformations of this procedure have evolved in the literature. For example, Miller and Blair (1985, p. 328) call the multipliers produced from this procedure "output-to-output" multipliers, and Szyrmer (1992) derives them from a "total-flow" perspective. As Miller and Blair (1985) point out, however, this approach is not valid in a situation where more than one sector's output is specified exogenously. 
sectoral detail, so they provide users with the ability to derive distributional estimates of indirect multiplier effects on specific sub-sectors.

One of the more important aspects of the output-based adjustment model presented here is its use in relative policy appraisals. The ability to predict the multiplier effects of policy-induced capacity constraints in a timely manner would allow regional decision makers to compare and contrast the outcomes of alternative management strategies prior to final implementation. Assessments such as this are especially important to federal and state resource management agencies that control resource extraction rates through adjustments in production constraints or quotas (e.g., fisheries, forestry, mining, and agricultural management agencies).

The output-based multipliers derived in this study measure only the short-run backward-linked impacts of predetermined output changes, however. The forward linkages due to the interconnectedness of the exogenized sector(s) to those sectors to which it sells its outputs are not explicitly considered. Certainly, this limitation warrants further consideration in empirical assessments of many capacity constraint scenarios, but while the backward linkage effect is relatively straightforward, unsatisfactory ways of interpreting and incorporating the direct effects of forward linkages may result in large distortions of the true estimates. Before the total distributional effects of sectoral policies that directly influence output can be properly evaluated, theoretical and empirical research in the future will have to find a way to incorporate both backward and forward linkage effects into economic impact models that contain a high level of sectoral detail.

\section{APPENDIX: A 3 × 3 EXAMPLE OF THE EQUIVALENCE BETWEEN THE MODIFIED OUTPUT-BASED MODEL AND THE MIXED EXOGENOUS/ ENDOGENOUS VARIABLES MODEL}

This Appendix corresponds to the example presented in Miller and Blair (1985, p. 328), which was used to demonstrate the mixed exogenous/endogenous variables approach. Suppose that we have a three-sector model and that the level of output of sector 3 is treated as exogenous. If $\mathrm{A}$ is assumed to equal

(A1) $A=\left[\begin{array}{lll}.15 & .25 & .30 \\ .20 & .05 & .18 \\ .20 & .20 & .10\end{array}\right]$

and the RPC for sector 3 is set to zero, then $A$ becomes

$$
\bar{A}=\left[\begin{array}{ccc}
.15 & .25 & .30 \\
.20 & .05 & .18 \\
0 & 0 & 0
\end{array}\right]
$$

and $(I-\bar{A})^{-1}$ would be given by 


$$
(I-\bar{A})^{-1}=\left[\begin{array}{ccc}
1.254 & 0.330 & 0.436 \\
0.264 & 1.122 & 0.281 \\
0 & 0 & 1
\end{array}\right]
$$

If $\hat{Y}_{3}$ is set equal to the change in $X_{3}$, assumed to be 150,000 , then the vector of output requirements as defined in equation (4) of the output-based approach can be solved as

$$
\left[\begin{array}{l}
X_{1} \\
X_{2} \\
X_{3}
\end{array}\right]=\left[\begin{array}{ccc}
1.254 & 0.330 & 0.436 \\
0.264 & 1.122 & 0.281 \\
0 & 0 & 1
\end{array}\right]\left[\begin{array}{c}
0 \\
0 \\
150,000
\end{array}\right] \text {, }
$$

which results in a solution vector of

$$
\left[\begin{array}{l}
X_{1} \\
X_{2} \\
X_{3}
\end{array}\right]=\left[\begin{array}{c}
65,346 \\
42,178 \\
150,000
\end{array}\right]
$$

To analyze the same three-sector example with the mixed exogenous/endogenous variables approach, equation (2) would take on the form

$$
\left[\begin{array}{ccr}
\left(1-a_{11}\right) & -a_{12} & 0 \\
-a_{21} & \left(1-a_{22}\right) & 0 \\
-a_{31} & -a_{32} & -1
\end{array}\right]\left[\begin{array}{c}
X_{1} \\
X_{2} \\
Y_{3}
\end{array}\right]=\left[\begin{array}{c}
\hat{Y}_{1}+a_{13} \hat{X}_{3} \\
\hat{Y}_{2}+a_{23} \hat{X}_{3} \\
-\left(1-a_{33}\right) \hat{X}_{3}
\end{array}\right]
$$

For this example, (A3) leads to

$$
\left[\begin{array}{rrr}
.85 & -.25 & 0 \\
-.20 & .95 & 0 \\
-.20 & -.20 & -1
\end{array}\right]\left[\begin{array}{c}
X_{1} \\
X_{2} \\
Y_{3}
\end{array}\right]=\left[\begin{array}{c}
\hat{Y}_{1}+45,000 \\
\hat{Y}_{2}+27,000 \\
-135,000
\end{array}\right]
$$

If the matrix of coefficients on the left-hand side of (A4) is set equal to $M$, then the inverse of $M$ is

$$
M^{-1}=\left[\begin{array}{rrr}
1.254 & .330 & 0 \\
.264 & 1.122 & 0 \\
-.304 & -.290 & -1
\end{array}\right]
$$

and 


$$
\left[\begin{array}{l}
X_{1} \\
X_{2} \\
Y_{3}
\end{array}\right]=\left[\begin{array}{rrr}
1.254 & .330 & 0 \\
.264 & 1.122 & 0 \\
-.304 & -.290 & -1
\end{array}\right]\left[\begin{array}{c}
\hat{Y}_{1}+45,000 \\
\hat{Y}_{2}+27,000 \\
-135,000
\end{array}\right]
$$

Assuming the changes in $\hat{Y}_{1}$ and $\hat{Y}_{2}$ are zero and the interest is only in the impact of exogenous change in output on other outputs, equation (A5) leads to

$$
\left[\begin{array}{l}
X_{1} \\
X_{2}
\end{array}\right]=\left[\begin{array}{l}
65,340 \\
42,174
\end{array}\right] \text {. }
$$

Except for rounding error, these values are the same as those generated for $X_{1}$ and $X_{2}$ in the solution vector represented by (A2).

\section{REFERENCES}

Eiser, D. and D. Roberts, 2002. "The Employment and Output Effects of Changing Patterns of Afforestation in Scotland," Journal of Agricultural Economics 53(1), 6581.

Findeis, J. and N. Whittlesey, 1984. "The Secondary Economic Impacts of Irrigation Development in Washington," Western Journal of Agricultural Economics 9, 233 243.

Ghosh, A., 1958. "Input-Output Approach to an Allocative System," Economica 25(1), 58-64.

Groenewold, N., A.J. Hagger, and J.R. Madden, 1987. "The Measurement of Industry Employment Contribution in an Input-Output Model," Regional Studies 21, 255-263.

Gruver, G.W., 1989. "On the Plausibility of Supply-Driven Input-Output Model: A Theoretical Basis for Input-Coefficient Change," Journal of Regional Science 29(3), 441-450.

Hoppe, R., 1978. "Building a Non-Metropolitan Input-Output Model: Minnesota's Region Six East," Agricultural Experiment Station, University of Minnesota, Technical Bulletin No. 313.

Jeong K, 1984. "The Relation Between Two Different Notions of Direct and Indirect Input Requirements," Journal of Macroeconomics 6, 473-476.

Johnson, T.G. and S.N. Kulshreshtha, 1982. "Exogenizing Agriculture in an Input-Output Model to Estimate Relative Impacts of Different Farm Types," Western Journal of Agricultural Economics 7(2), 187-198.

Lahr, M.L. and R.E. Miller, 2001. "A Taxonomy of Extractions," in M.L. Lahr and R.E. Miller (editors), Regional Science Perspectives in Economic Analysis. Elsevier Science, Amsterdam.

Leung, P.S. and S. Pooley, 2002. "Regional Economic Impacts of Reductions in Fisheries Production: A Supply-Driven Approach," Marine Resource Economics 16(4), 251262. 
Marcouiller, D.W., D.F. Schreiner, and D.K. Lewis, 1996. "The Impact of Forest Land Use on Regional Value Added," The Review of Regional Studies 26, 211-233.

Miller, R.E. and P.D. Blair, 1985. Input-Output Analysis: Foundations and Extensions. Prentice-Hall, London.

Natcher, B., J. Greenberg, and M. Herrmann, 1999. "Impact Analysis of Changes in Fishery Regulations in the Norton Sound Red King Crab Fishery," Arctic 52(1), 3-39.

National Marine Fisheries Service, 2001. "Final Environmental Impact Statement, Fishery Management Plan for the Pelagic Fisheries of the Western Pacific Region," 2 vols.

Oosterhaven, J., 1988. "On the Plausibility of the Supply-Driven Input-Output Model," Journal of Regional Science 28(2), 203-217.

Oosterhaven, J., 1989. "The Supply-Driven Input-Output Model: A New Interpretation but still Implausible," Journal of Regional Science 29(3), 459-465.

Papadas, C.T. and D.C. Dahl, 1999. "Supply-Driven Input-Output Multipliers," Journal of Agricultural Economics 50(2), 269-285.

Petkovich, M.D. and C.T.K. Ching, 1978. "Modifying a One Region Leontief InputOutput Model to Show Sector Capacity Constraints," Western Journal of Agricultural Economics 3, 173-179.

Ritz, P.M. and E. Spaulding, 1975. "Unpublished Memorandum on Basic Input-Output Terminology," Department of Commerce, Bureau of Economic Analysis, Interindustry Economics Division, February 25.

Roberts, D., 1994. "A Modified Leontief Model for Analysing the Impact of Milk Quotas on the Wider Economy," Journal of Agricultural Economics 45(1), 90-101.

Szyrmer, J.M., 1992. "Input-Output Coefficients and Multipliers from a Total-Flow Perspective," Environment and Planning A 24, 921-937.

Tanjuakio, R.V., S.E. Hastings, and P.J. Tytus, 1996. "The Economic Contribution of Agriculture in Delaware," Agricultural and Resource Economics Review 25(1), 4653.

Waters, E.C., D.W. Holland, and B.A. Weber, 1994. "Interregional Effects of Reduced Timber Harvests: The Impact of the Northern Spotted Owl Listing in Rural and Urban Oregon," Journal of Agricultural and Resource Economics 19(1), 141-160. 